\title{
基于煤萃取物的类石墨状多孔炭的制备及其 电容性能研究
}

\author{
李金宏 ${ }^{1}$, 周岐雄 ${ }^{1}$, 米红宇 ${ }^{1}$, 李 显 $^{2}$, 李惠萍 ${ }^{1}$
}

(1. 新疆大学 新疆维吾尔自治区洁净煤转化与化工过程重点实验室, 乌鲁木齐 830046; 2. 华中科技大学 煤燃烧 国家重点实验室, 武汉 430074)

摘 要: 以褐煤萃取物为炭前驱体, $\mathrm{MgO}$ 为阻隔剂, $\mathrm{KOH}$ 为活化剂, 在 $800^{\circ} \mathrm{C}$ 惰性气氛下制备出类石墨状多孔炭材 料。对该多孔炭材料进行了红外(FTIR)、X 射线衍射(XRD)、透射电镜(TEM)和拉曼(Raman)表征。以活化前和活 化后多孔炭为电极材料, 利用循环伏安、恒电流充放电、交流阻抗对其进行了电化学电容性能评价和比较。结果表 明: 活化后炭材料呈现多孔的薄膜状, 比表面积高达 $1396 \mathrm{~m}^{2} / \mathrm{g}$, 而活化前炭材料比表面积仅为 $138.4 \mathrm{~m}^{2} / \mathrm{g}$ 。当电流 密度为 $1 \mathrm{~A} / \mathrm{g}$ 和 $4 \mathrm{~A} / \mathrm{g}$ 时, 活化后炭材料比电容分别为 $533 \mathrm{~F} / \mathrm{g}$ 和 $390 \mathrm{~F} / \mathrm{g}$; 而活化前炭材料对应的比电容为 $366 \mathrm{~F} / \mathrm{g}$ 和 $198 \mathrm{~F} / \mathrm{g}$ 。在电流密度为 $5 \mathrm{~A} / \mathrm{g}$ 下循环 8000 圈后，活化前后炭材料的电容保持率分别为 $72.5 \%$ 和 $89.6 \%$ 。可见，经 过 $\mathrm{KOH}$ 活化后的炭材料比电容和电化学稳定性有了显著提高。该研究证明阻隔剂和活化剂的使用, 能够获得高度 柔性的高电容性能的类石墨状多孔炭。

关 键 词: 煤萃取物; 多孔炭; 电极材料; 电容性能

中图分类号: TQ174 文献标识码: A

\section{Preparation and Capacitive Properties of Graphite-like Porous Carbon Based on Coal Extracts}

\author{
LI Jin-Hong ${ }^{1}$, ZHOU Qi-Xiong ${ }^{1}$, MI Hong-Yu ${ }^{1}$, LI Xian ${ }^{2}$, LI Hui-Ping ${ }^{1}$
}

(1. Xinjiang Uygur Autonomous Region Key Laboratory of Coal Clean Conversion \& Chemical Engineering Process, Xinjiang University, Urumchi 830046, China; 2. State Key Laboratory of Coal Combustion, Huazhong University of Science and Technology, Wuhan 430074, China)

\begin{abstract}
By using extracts of lignites as carbon precursors, $\mathrm{MgO}$ as isolation agent, and $\mathrm{KOH}$ as activator, porous graphene-like carbon materials were prepared at $800^{\circ} \mathrm{C}$ under an inert atmosphere. Porous carbon materials were characterized by FTIR, XRD, TEM and Raman. Further, electrochemical capacitive properties of porous carbons before and after activation as the electrodes were evaluated by cyclic voltammetry, galvanostatic charge-discharge and impedance spectroscopic techniques. Results indicate that carbon materials after activation exhibit porous film-like shape. The specific surface areas of carbon materials before and after activation are $138.4 \mathrm{~m}^{2} / \mathrm{g}$ and $1396 \mathrm{~m}^{2} / \mathrm{g}$, respectively. Specific capacitances of carbon materials after activation reach $533 \mathrm{~F} / \mathrm{g}$ and $390 \mathrm{~F} / \mathrm{g}$ at $1 \mathrm{~A} / \mathrm{g}$ and $4 \mathrm{~A} / \mathrm{g}$, respectively, while those of carbon materials before activation at same conditions are $366 \mathrm{~F} / \mathrm{g}$ and $198 \mathrm{~F} / \mathrm{g}$. After 8000 cycles at a current density of $5 \mathrm{~A} / \mathrm{g}$, the capacitance retentions of carbon materials before and after activation are $72.5 \%$ and
\end{abstract}

收稿日期：2015-07-16; 收到修改稿日期：2015-09-18

基金项目：新疆维吾尔自治区科技厅国际科技合作计划项目(20136007)

Xinjiang Uygur Autonomous Region Science and Technology Department of International Science and Technology Cooperation Plan Project (20136007)

作者简介: 李金宏(1988-), 男, 硕士研究生. E-mail: 978499728@qq.com

通讯作者: 周岐雄, 副教授. E-mail: qixiong42@126.com 
$89.6 \%$, respectively. Accordingly, after $\mathrm{KOH}$ activation, the specific capacitance and electrochemical stability of carbon materials are significantly improved. This study verifies that the use of the isolation agent and the activator can obtain highly flexible graphene-like porous carbon with high capacitance performance.

Key words: coal extracts; porous carbon; electrode material; capacitive performance

双电层电容器具有充电时间短、循环效率高、 使用寿命长、温度特性好和绿色环保等优点, 因此 在电动车辆、通讯设备、航空航天和国防科技等领 域得到广泛应用 ${ }^{[1-3]}$ 。但是双电层电容器存在电容较 低的问题, 这大大限制了其在车辆和战车的启动电 源、激光武器的脉冲能源等高功率场合的应用。因 此, 发展先进的电极材料成为开发高性能电容器的 重要课题之一。

多孔炭材料具有原料来源丰富、制作过程简单、 比表面积高和电容特性良好等优点, 成为双电层电 容器较为理想的电极材料。近年来, 以煤为炭前驱 体制备双电层电容器用电极材料备受关注, 因为这 不仅可以实现煤的多级利用、增加其附加值、减少 环境污染等, 而且为高性能的环保储能元件提供了 材料保证。Kierzek 等 ${ }^{[4]}$ 以高挥发性烟煤、半焦和沥 青等为原料, $\mathrm{KOH}$ 为活化剂, 制备出比电容为 200 320 F/g 的活性炭电极材料; $\mathrm{He}$ 等 ${ }^{[5]}$ 以石油焦为 炭源, 采用 $\mathrm{KOH}$ 活化法制备出的电极材料最大比 电容为 $298.8 \mathrm{~F} / \mathrm{g}$, 经过 200 次充放电循环后, 比电 容仍保持在 $279.6 \mathrm{~F} / \mathrm{g}$ 。Zhang 等 ${ }^{[6]}$ 以烟煤为原料, 采 用 $\mathrm{KOH}$ 快速活化法制备出比电容为 $370 \sim 270 \mathrm{~F} / \mathrm{g}$ 的 富氧活性炭电极材料。这些研究显示, 煤基炭材料 通过 $\mathrm{KOH}$ 活化处理有望得到高性能的双电层电容 器电极材料。

新疆煤炭资源丰富, 且煤种齐全。为了实现新 疆煤炭的高效综合利用和开发新型储能器件用关键 材料, 本研究以新疆褐煤为起始原料, 首先对其进 行热萃取法提质, 以获得高含碳量、富含芳环的褐 煤萃取物; 然后, 以煤萃取物为炭源, 利用 $\mathrm{MgO}$ 的 阻隔功能, 通过 $\mathrm{KOH}$ 活化, 制备出柔性的、高比表 面积的薄膜状多孔炭, 并对其进行电化学性能评价。

\section{1 实验方法}

实验所用的煤为新疆淖毛湖褐煤, 1-甲基菜、浓 盐酸 $(\mathrm{HCl})$ 、浓硫酸 $\left(\mathrm{H}_{2} \mathrm{SO}_{4}\right)$ 、氢氧化钾 $(\mathrm{KOH})$ 、氧化 镁 $(\mathrm{MgO}) 、 乙$ 醇 $\left(\mathrm{CH}_{3} \mathrm{CH}_{2} \mathrm{OH}\right) 、 \mathrm{~N}, \mathrm{~N}-$ 二甲基甲酰胺 (DMF)均为分析纯, 使用的水为去离子水。

\section{1 多孔炭制备}

将 250 841 $\mu \mathrm{m}$ 的煤粉及 1-甲基萗萃取剂的混合
物置于自制的间歇式萃取釜中, 程序升温至 $350^{\circ} \mathrm{C}$, 升温速率为 $5^{\circ} \mathrm{C} / \mathrm{min}$, 并在 $350^{\circ} \mathrm{C}$ 保持 $1 \mathrm{~h}$, 完成萃取 过程。然后, 釜温降至室温后, 将混合物分离。最终, 萃取物的收率为 $32.33 \mathrm{wt} \%$ (干燥无灰基, daf), 用此 萃取物作为制备多孔炭的炭源。

$\mathrm{MgO}$ 、萃取物和 $\mathrm{DMF}$ 按质量比为 $1: 20: 40$ 加入烧杯中搅拌过夜, 然后将该混合液置于 $120^{\circ} \mathrm{C}$ 鼓风干燥箱中干燥; 再将其放入氮气气氛的管式炉 中, 升温至 $350^{\circ} \mathrm{C}$, 保持 $1 \mathrm{~h}$; 继续升温至 $800^{\circ} \mathrm{C}$, 保 持 $3 \mathrm{~h}$, 升温速率为 $5^{\circ} \mathrm{C} / \mathrm{min}$ 。炭化后的产物用 $3 \mathrm{~mol} / \mathrm{L} \mathrm{HCl}$ 反复浸泡以除去 $\mathrm{MgO}$, 之后用大量去 离子水洗涤以去除 $\mathrm{Mg}^{2+}$ 和 $\mathrm{Cl}^{-}$。最后, 产物用 $120^{\circ} \mathrm{C}$ 鼓风干燥 $8 \mathrm{~h}$ 得到 “活化前多孔炭”, 记为 $\mathrm{C} 1$, 其 相对于萃取物的收率为 $44.96 \mathrm{wt} \%$ (daf)。

为了更一步丰富材料孔结构和提高其比表面积, 对活化前多孔炭进行 $\mathrm{KOH}$ 活化处理。将活化前多

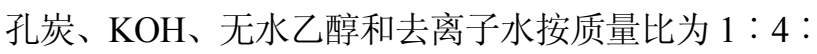
5:10 加入烧杯中搅拌过夜, $120^{\circ} \mathrm{C}$ 鼓风干燥该混合 物。然后, 将混合物在 $800^{\circ} \mathrm{C} 、 \mathrm{~N}_{2}$ 气氛下的管式炉 中活化 $3 \mathrm{~h}$, 升温速率为 $5^{\circ} \mathrm{C} / \mathrm{min}$ 。将活化后的混合 物用 $1 \mathrm{~mol} / \mathrm{L} \mathrm{HCl}$ 浸泡后, 用去离子水清洗至滤液 为中性, 再将其用 $120^{\circ} \mathrm{C}$ 鼓风干燥 $8 \mathrm{~h}$ 即得到 “活化 后多孔炭”, 记为 $\mathrm{C} 2$, 其相对于活化前多孔炭的收 率为 $53.38 \mathrm{wt} \%$ (daf)。

为了对比, 在不加 $\mathrm{MgO}$ 的条件下, 用制备活化 前多孔炭的方法处理褐煤萃取物, 得到 “直接炭化 物”, 记为 $\mathrm{C} 3$ 。

\section{2 电极制备}

将多孔炭、乙炔黑和聚四氟乙烯按质量比为 8: $1: 1$ 混合均匀后压制成薄片状材料; 然后, 将该薄 片在 $80^{\circ} \mathrm{C}$ 真空干燥箱干燥 $24 \mathrm{~h}$ 后, 裁成质量为 $1.5 \mathrm{mg}$ 的电极片(活性物质的质量为 $1.2 \mathrm{mg}$ ); 最后, 将电极片压在炭纸(作为集流体)上, 即得到所需工 作电极。测试前将工作电极在电解液中浸泡过夜, 电化学测试采用饱和甘录电极为参比电极、铂电极 为对电极的三电极体系, 所用的电解液为 $1 \mathrm{~mol} / \mathrm{L}$ $\mathrm{H}_{2} \mathrm{SO}_{4}$ 溶液。

\section{3 样品表征}

采用德国 Elementar 公司 VarioELIII型元素分析 
仪对样品进行元素分析; 利用美国 TA 公司 SDTQ600 型热重分析仪对样品进行热重分析(TG-DTG); 利用德国布鲁克 VERTEX70 型红外光谱仪测试样 品的红外光谱(FTIR)。采用德国布鲁克 D8 型 X 射线衍射仪测试样品的结构, 实验条件: $\mathrm{CuK} \alpha$ 辐 射 $(\lambda=0.154056 \mathrm{~nm})$, 扫描范围 $2 \theta=10^{\circ} \sim 80^{\circ}$ 。采用 德国 BPUKER 公司的激光显微共聚焦拉曼光谱仪 测试样品的拉曼光谱 (Raman); 采用日本日立 Hitachi-600 型透射电镜对样品的微观形貌进行表征 (TEM)。采用美国康塔仪器公司的全自动比表面及孔 径分析仪 ASIQM0002-6 在 $77 \mathrm{~K}$ 下测试样品对氮气 的吸附和脱附等温线, 由 Brunauer-Emmett-Teller (BET) 法计算比表面积, 用 Density-FunctionalTheory (DFT) 模型拟合吸附等温线得到孔结构。采 用上海辰华 CHI660 电化学工作站测试样品的电化 学性能, 采用武汉蓝电 CT2001A 电池测试系统测试 样品的循环寿命。

\section{2 结果与讨论}

\section{1 萃取物的工业分析、元素分析及热重分析} 煤萃取物的工业分析和元素分析结果如表 1 所 示。由表可知, 萃取物显著特征是挥发分高 $(75.29 \mathrm{wt} \%$, $\mathrm{daf})$, 碳含量高 $(77.6 \mathrm{wt} \%$, daf)。图 1 是萃取物的热重 曲线, 在室温 $380^{\circ} \mathrm{C}$ 之间萃取物失重 $11.18 \%$, 主要
是水分和挥发分的脱除所致，失重较慢; 在 380 $600^{\circ} \mathrm{C}$ 之间失重 $51.24 \%$, 主要是有机物的快速热解 所致, 失重最快。随着热解反应的进行, 样品逐渐进 入炭化阶段, 失重变得缓慢, 此阶段主要发生半焦 化反应、脱氢反应和缩聚反应，最后生成焦炭。由 图 1 可知, 煤萃取物在 $800^{\circ} \mathrm{C}$ 之后热失重基本不变, 因此, $800^{\circ} \mathrm{C}$ 可以作为煤萃取物炭化温度。

\section{2 萃取物和多孔炭材料的 FTIR 分析}

图 2 是萃取物和活化后多孔炭(C2)的 FTIR 图谱, 由图可见, 萃取物具有明显的吸收峰。萃取物在 $3430 \mathrm{~cm}^{-1}$ 处的吸收峰为 $\mathrm{O}-\mathrm{H}$ 伸缩振动峰; 2920 和 $2850 \mathrm{~cm}^{-1}$ 处的峰为脂肪族的 $\mathrm{C}-\mathrm{H}$ 伸缩振动峰; 1600 和 $1440 \mathrm{~cm}^{-1}$ 附近的峰均为芳环上 $\mathrm{C}=\mathrm{C}$ 的伸缩振动 峰; 1300 1100 $\mathrm{cm}^{-1}$ 之间的峰是脂肪、醇、醚、酚、 酮类物质的 $\mathrm{C}-\mathrm{O}$ 伸缩振动峰; $820 \sim 770 \mathrm{~cm}^{-1}$ 之间的 峰为芳环平面外的 $\mathrm{C}-\mathrm{H}$ 弯曲振动峰 ${ }^{[7-10]}$ 。活化后多 孔炭 $(\mathrm{C} 2)$ 在 $3430 \mathrm{~cm}^{-1}$ 处存在较明显吸收峰, 对应于 $\mathrm{O}-\mathrm{H}$ 伸缩振动峰; 在 $1630 \mathrm{~cm}^{-1}$ 处的吸收峰为 $\mathrm{C}=\mathrm{O}$ 伸缩振动峰; 1570 和 $1410 \mathrm{~cm}^{-1}$ 处附近的峰为 $\mathrm{COOH}$ 的伸缩振动峰; 在 $1385 \mathrm{~cm}^{-1}$ 附近的峰为 $\mathrm{C}-\mathrm{H}$ 伸缩 振动峰, $1080 \mathrm{~cm}^{-1}$ 附近的吸收峰为含 $\mathrm{C}-\mathrm{O}$ 基团化合 物的伸缩振动峰 ${ }^{[1-16]}$ 。通过对比, 可以发现活化后 多孔炭的谱图中萃取物的特征吸收峰显著减弱, 说 明经过高温炭化和进一步高温活化后，萃取物能够 较为完全地转变成炭材料。

表 1 萃取物的工业分析和元素分析

Table 1 Proximate and ultimate analysis of extracts

\begin{tabular}{|c|c|c|c|c|c|c|c|c|c|c|}
\hline \multicolumn{4}{|c|}{ Proximate analysis / wt $\%$} & \multicolumn{5}{|c|}{ Ultimate analysis $/(\mathrm{wt} \%, \mathrm{daf})$} & \multirow{2}{*}{$\mathrm{H} / \mathrm{C}$} & \multirow{2}{*}{$\mathrm{O} / \mathrm{C}$} \\
\hline$M_{\mathrm{ad}}$ & $A_{\text {ad }}$ & $V_{\text {daf }}$ & $F C_{\text {ad }}$ & $\mathrm{C}$ & $\mathrm{H}$ & $\mathrm{N}$ & $\mathrm{S}$ & $\mathrm{O}^{*}$ & & \\
\hline 1.76 & 0.93 & 75.29 & 24.05 & 77.6 & 5.76 & 0.99 & 0.13 & 15.52 & 0.89 & 0.15 \\
\hline
\end{tabular}

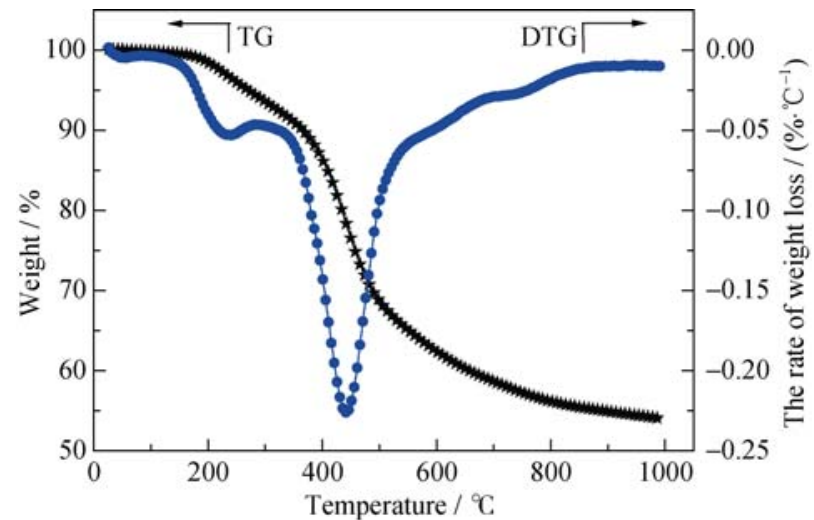

图 1 萃取物的热重曲线

Fig. 1 TG-DTG curves of the extracts

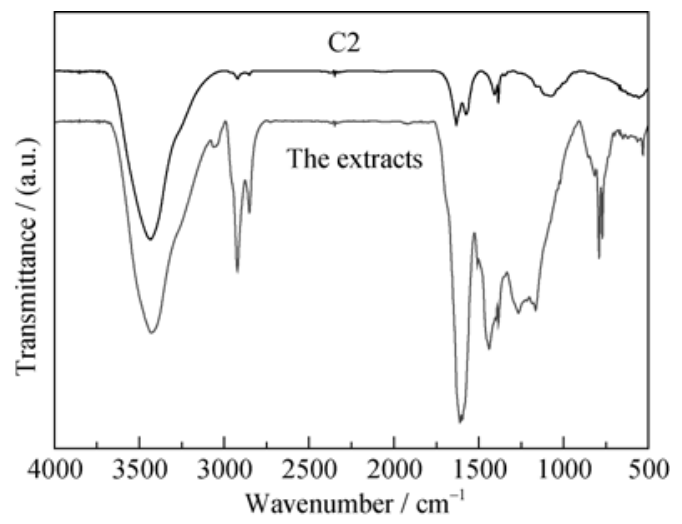

图 2 萃取物和多孔炭(C2)的 FTIR 图谱

Fig. 2 FTIR spectra of the extracts and C2 


\section{3 多孔炭材料的 XRD 和 Raman 分析}

图 3 是活化后多孔炭(C2)的 XRD 谱图(a)和 Raman 谱图(b)。从图 3(a)中可以观察到, 在 $2 \theta=25^{\circ}$ 和 $42.5^{\circ}$ 附近出现了较强的衍射峰和很弱的衍射峰, 分 别对应于石墨的(002)晶面和(100)晶面。这两个峰呈弥 散状且强度较弱, 类似于无定形石墨结构的谱图 ${ }^{[17-20]}$, 表明制备的多孔炭主要以无定型形式存在。图 3(b) 的 Raman 谱图在 1334(D 峰)和 $1580 \mathrm{~cm}^{-1}$ ( $\mathrm{G}$ 峰)处出 现两个峰，前者对应于碳原子 $\mathrm{sp}^{3}$ 杂化的特征峰，反 映了碳原子的无序性 ${ }^{[21]}$; 后者对应于碳原子 $\mathrm{sp}^{2}$ 杂 化的特征峰, 反映了碳原子的有序性 ${ }^{[21]}$ 。在 1334 和 $1580 \mathrm{~cm}^{-1}$ 处特征峰的强度比 $\left(I_{\mathrm{D}} / I_{\mathrm{G}}\right)$ 为 1.03 , 表明该 炭材料结构主要以无序形式存在 ${ }^{[22]}$ 。

\section{4 多孔炭材料的微观结构分析}

图 4(a c) 为不同条件下制备的多孔炭的 TEM 照片。从图 4(a)中看出, 直接炭化物(C3)具有较厚的 块状结构。图 4(b)为活化前多孔炭(C1)的 TEM 照片, 当引入 $\mathrm{MgO}$ 并炭化后, 样品变薄, 且出现一些明显 的孔洞, 这是 $\mathrm{MgO}$ 去除所形成的, 说明 $\mathrm{MgO}$ 具有 造孔功能, 而且能够阻止样品炭层的紧密堆积, 使 其容易形成薄层结构。图 4(c)为活化后多孔炭(C2), 经过 $\mathrm{KOH}$ 活化后样品呈现出相互连通的薄膜网状 结构, 并且存在大量的孔洞, 类似于天然石墨烯剥
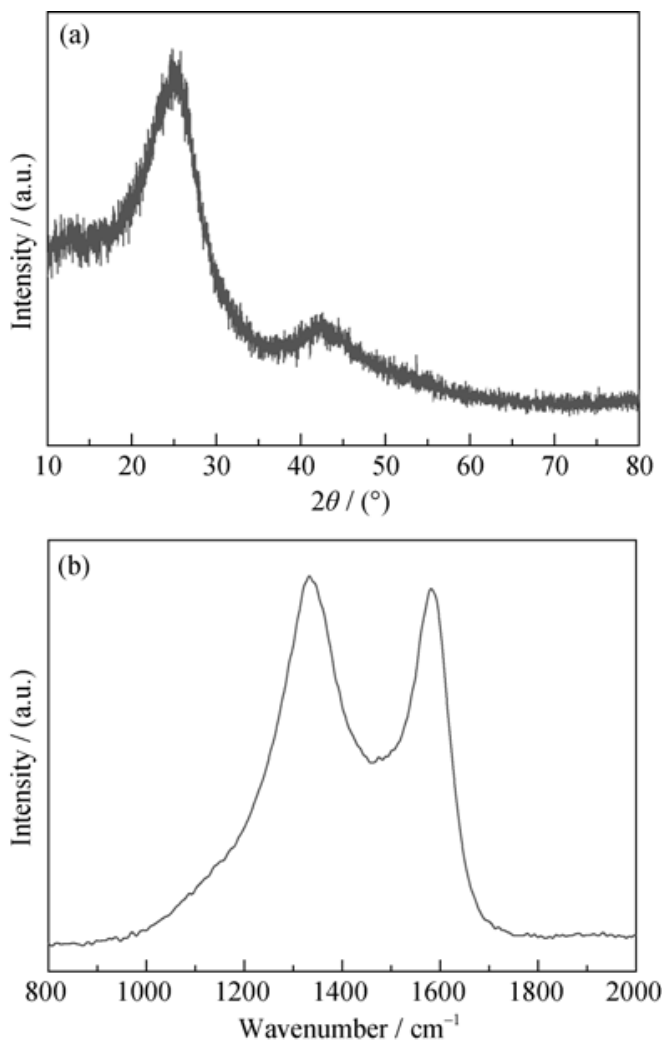

图 $3 \mathrm{C} 2$ 的 XRD 谱图(a) 和 Raman 谱图(b)

Fig. 3 XRD spectra (a) and Raman spectrum (b) of C2
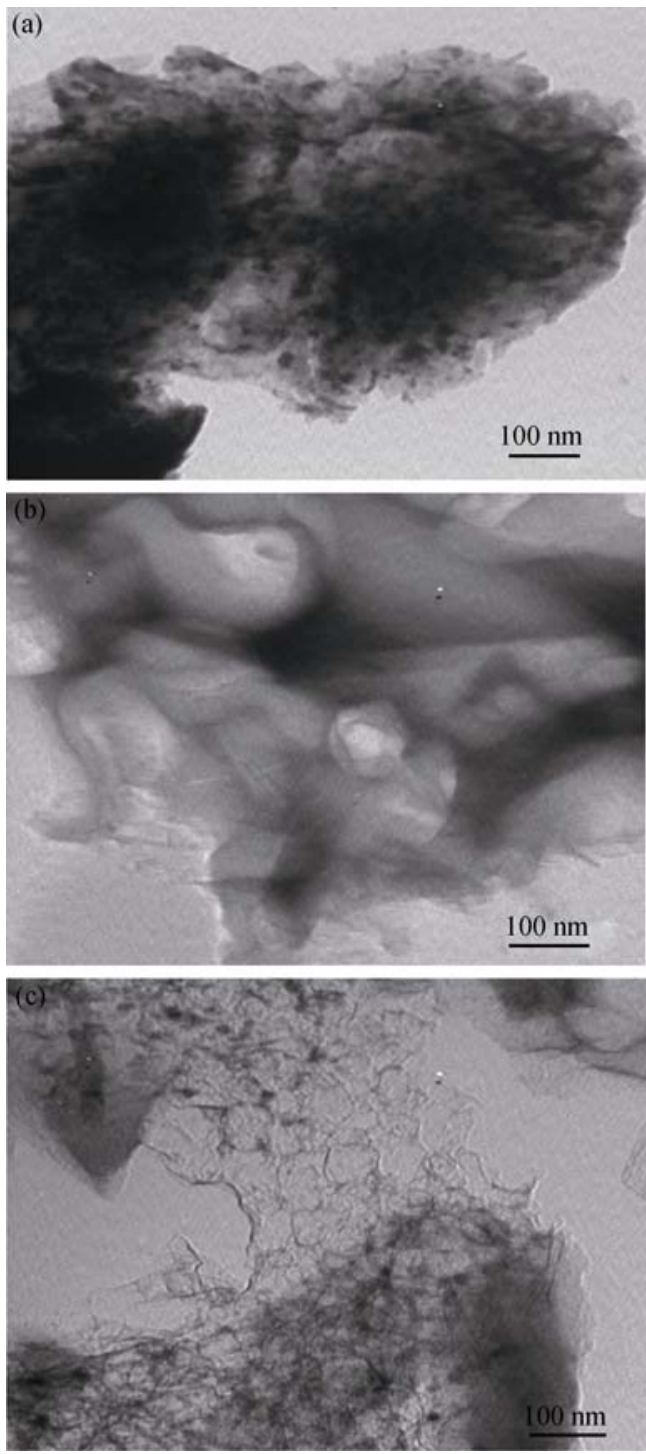

图 4 直接炭化物 $\mathrm{C} 3(\mathrm{a})$ 、活化前多孔炭 $\mathrm{C} 1(\mathrm{~b})$ 和活化后多孔 炭 $\mathrm{C} 2$ (c) 的 TEM 照片

Fig. 4 TEM images of C3 (a), C1 (b) and C2 (c)

离后的柔性薄膜形状, 这种结构有助于电化学过程 中电解液快速渗透, 并且与电极充分接触。

\section{5 多孔炭材料的 $\mathrm{N}_{2}$ 吸脱附测试}

图 5(a,b)分别为活化前 $(\mathrm{C} 1)$ 和活化后 $(\mathrm{C} 2)$ 多孔 炭在 $77 \mathrm{~K}$ 下的 $\mathrm{N}_{2}$ 吸脱附等温线图, 插图是采用 DFT 算法得到的相应孔径分布图。图 5(a)、(b)显示 活化前后样品的吸附等温线均为 IV 型特征曲线。当 相对压力在 0.4 0.95 之间时, 它们均存在脱附滞后 现象，说明材料中存在大量的微孔。活化前电极材 料的吸附量远小于活化后的吸附量, 由此推测活化 前电极材料的比表面积小于活化后的。采用 BET 法 得到活化前后多孔炭的比表面积分别为 138.4 和 $1396 \mathrm{~m}^{2} / \mathrm{g}$, 总孔容分别为 0.078 和 $0.690 \mathrm{~cm}^{3} / \mathrm{g}$ 。这 些数据说明, $\mathrm{KOH}$ 活化后, 炭材料的比表面积和孔 体积得到大幅提高, 这与文献[23-24]的结果一致。 

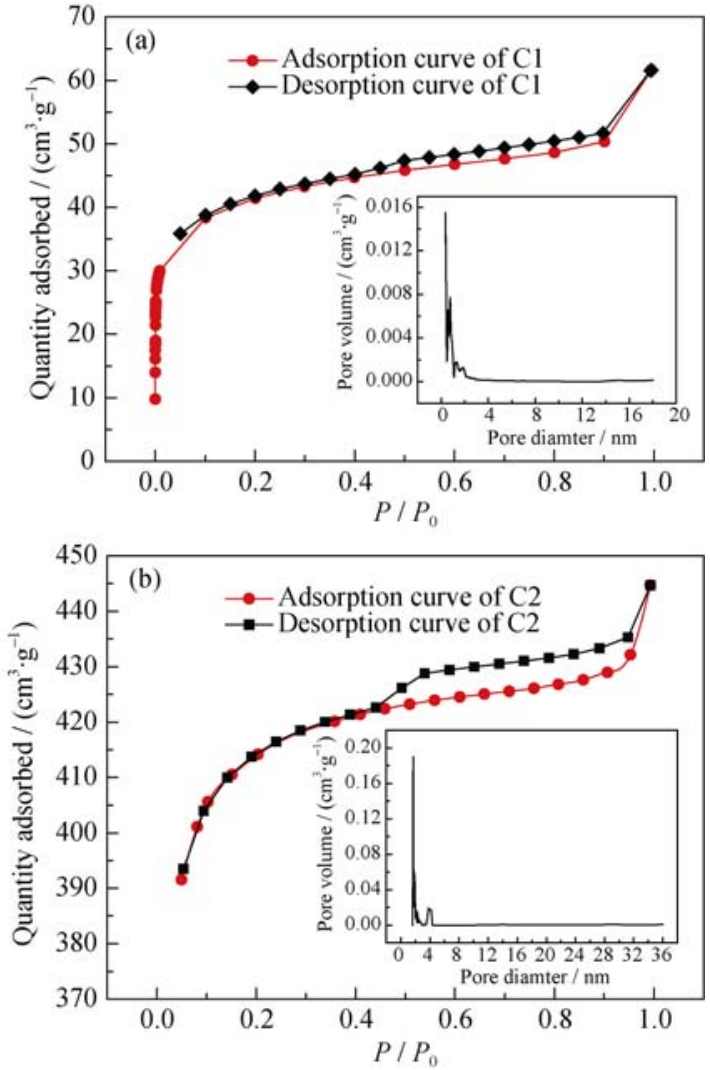

图 $577 \mathrm{~K}$ 下 $\mathrm{C} 1(\mathrm{a})$ 和 $\mathrm{C} 2(\mathrm{~b})$ 的 $\mathrm{N}_{2}$ 吸脱附等温线及其孔径分布 曲线

Fig. $5 \mathrm{~N}_{2}$ adsorption-desorption isotherms and pore size distribution of $\mathrm{C} 1$ (a) and $\mathrm{C} 2$ (b)

从孔径分布图(插图)可以看到, 活化前样品的孔径 小于 $2 \mathrm{~nm}$, 样品主要存在微孔; 而活化后样品的孔 径集中在 1.6 2.1 nm, 少量孔径达到 $4 \mathrm{~nm}$ 左右, 这 表明样品经活化后，不仅产生大量微孔，还形成了 少量介孔。有研究表明, 多孔炭材料作为双电层电 容器用电极材料的电化学性能不仅取决于多孔炭材 料的比表面积, 还取决于其孔容及孔结构分布 ${ }^{[25]}$ 。

一般地, 具有较高比表面积和孔径分布合适的炭电 极材料能够表现出良好的电化学性能 ${ }^{[26]}$ 。

\section{6 电极材料的循环伏安测试}

图 6(a,b)分别为活化前多孔炭 $(\mathrm{C} 1)$ 和活化后多孔 炭 $(\mathrm{C} 2)$ 电极材料在 $1 、 2 、 3 、 5 、 10 \mathrm{mV} / \mathrm{s}$ 扫速下的循 环伏安 $(\mathrm{CV})$ 曲线，电位区间为 $0 \sim 0.8 \mathrm{~V}$ 。由图 6(a)、(b) 可见, 这些曲线都近似于矩形, 显示出双电层电容特 性。对比发现，同一扫速下，活化前样品的 CV 曲线所 围面积小于活化后样品的 CV 曲线所围面积, 由此推 测活化前多孔炭电极的电容可能小于活化后的。另外, 随着扫速的提高, $\mathrm{CV}$ 曲线形状基本未改变, 表现出良 好的电化学稳定性。观察 $\mathrm{CV}$ 曲线的两端可以发现, 当 扫描速率反向时, 有瞬时反向电流产生, 表明电极内 阻很小, 具有良好的电化学可逆性 ${ }^{[27]}$ 。
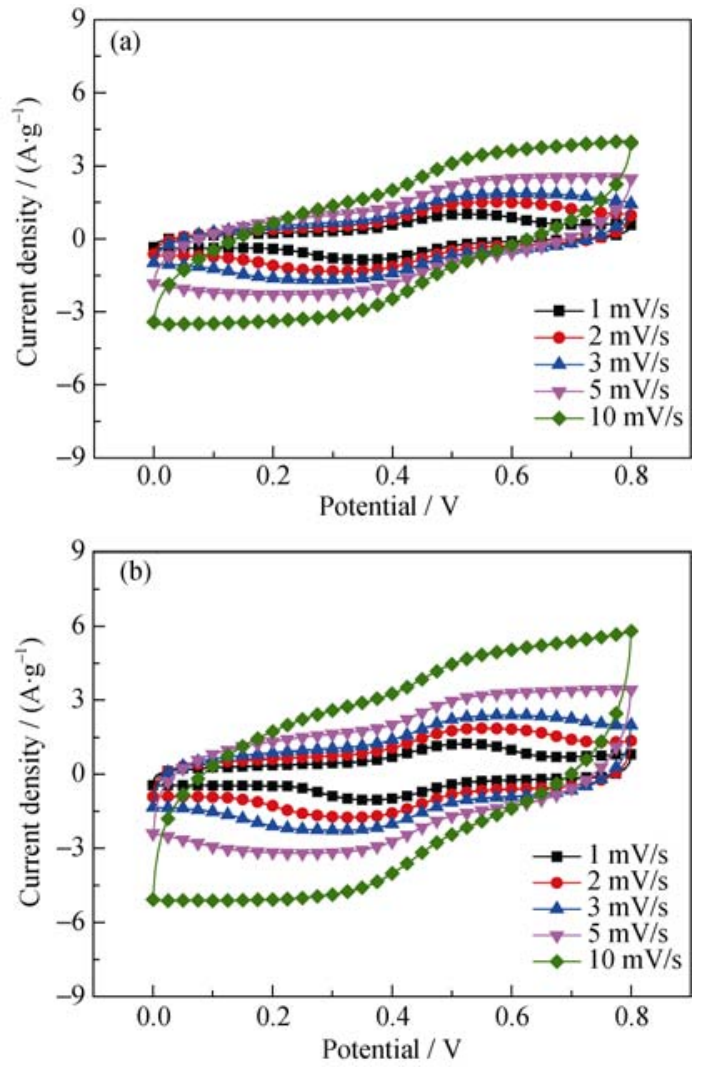

图 $6 \mathrm{C} 1(\mathrm{a})$ 和 $\mathrm{C} 2(\mathrm{~b})$ 电极在不同扫速下的循环伏安曲线

Fig. 6 Cyclic voltammograms of C1 (a) and C2 (b) electrodes at different scanning rates

\section{7 电极材料的充放电测试}

图 7(a,b)分别为活化前多孔炭 $(\mathrm{C} 1)$ 和活化后多 孔炭 $(\mathrm{C} 2)$ 电极材料在不同电流密度 $(1 、 2 、 3 、 4 \mathrm{~A} / \mathrm{g})$ 下的恒流充放电 $(C P)$ 曲线。图 7(a)、(b)显示 CP 曲 线呈现较好的镜面对称性, 表明两个电极材料充放 电效率较高, 电化学的可逆性良好, 这与 $\mathrm{CV}$ 曲线 结果相吻合。此外, 在同一电流密度下, 活化前炭材 料的放电时间要少于活化后的，这意味着活化前的 比电容小于活化后的。它们的比电容能够用下面的 公式计算:

$$
C=\frac{I \Delta t}{m \Delta V}
$$

式中 $C$ 为比电容 $(\mathrm{F} / \mathrm{g}), I$ 为恒流放电电流 $(\mathrm{A}), \Delta V$ 为 电位区间 $(\mathrm{V}), \Delta t$ 为放电时间 $(\mathrm{s}), m$ 为单电极质量 $(\mathrm{g})$ 。通过计算, 活化前多孔炭电极在电流密度为 1 、 $2 、 3 、 4 \mathrm{~A} / \mathrm{g}$ 下的比电容分别为 366、322、252、 $197 \mathrm{~F} / \mathrm{g}$ ，电容保持率为 $54 \%$; 活化后多孔炭电极比 电容分别 533、492、448、390 F/g，电容保持率为 $73 \%$, 正如图 8(a)、(b)所示。在这里, 比电容随着电 流密度的增加而呈现下降趋势, 这可能是由于电流 增加导致电极体相未被有效利用所致 ${ }^{[28]}$ 。尽管如此, 活化后多孔炭材料仍表现出相当高的比电容, 

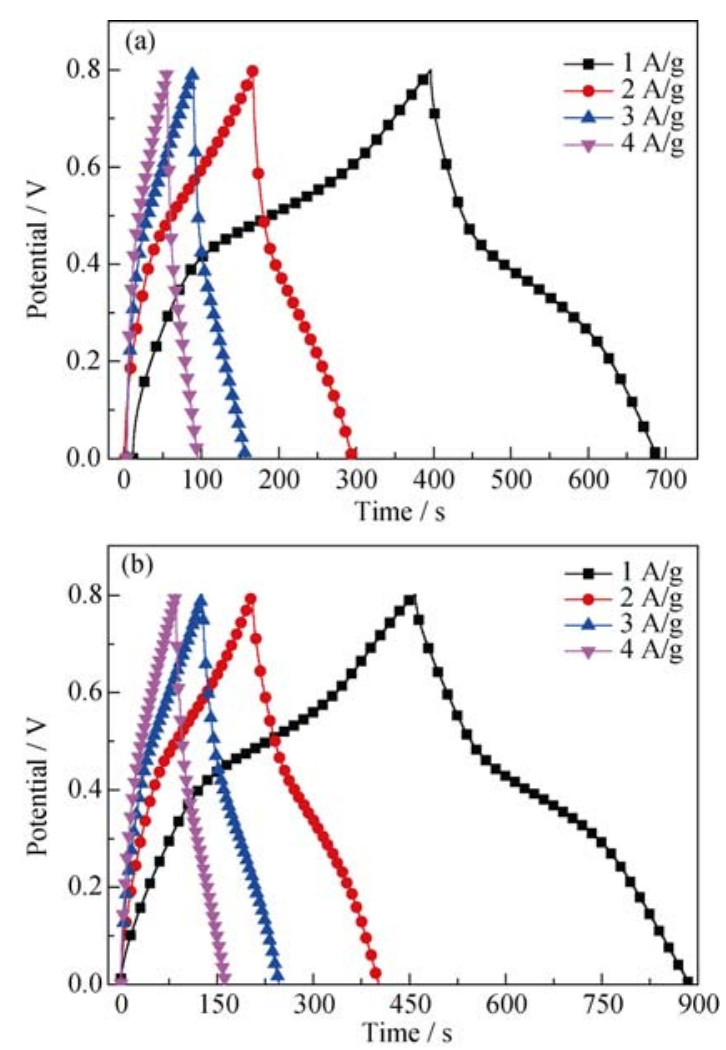

图 $7 \mathrm{C} 1(\mathrm{a})$ 和 $\mathrm{C} 2(\mathrm{~b})$ 电极在不同电流密度下的充放电曲线 Fig. 7 Charge/discharge curves of C1 (a) and C2 (b) electrodes at different current densities
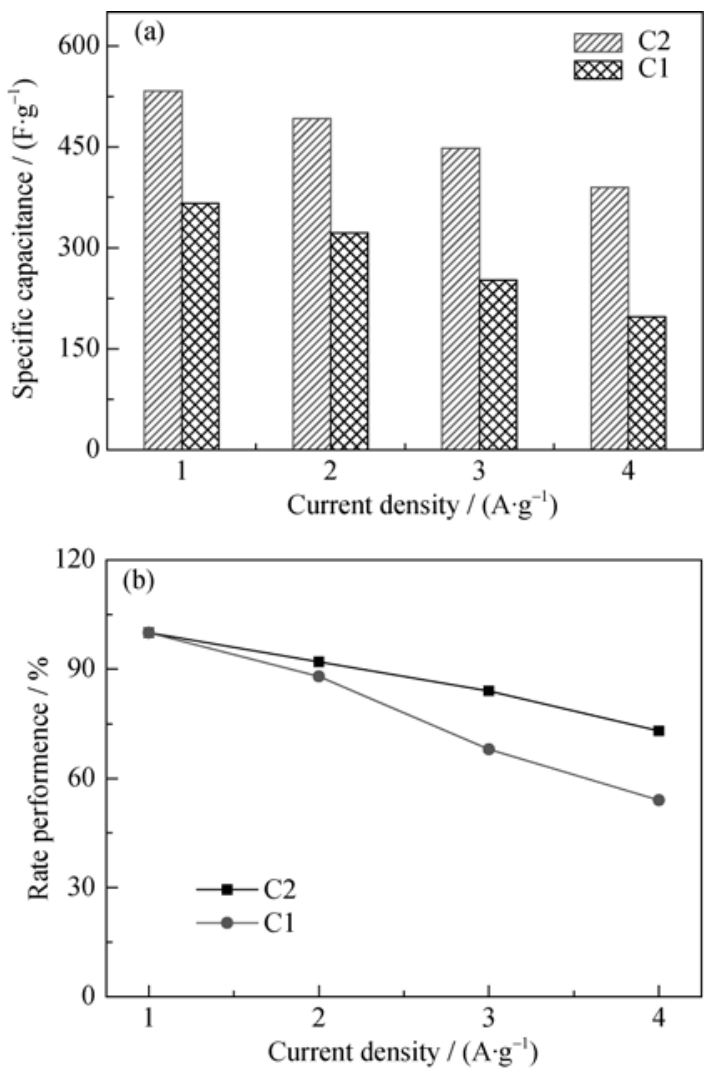

图 $8 \mathrm{C} 1$ 和 $\mathrm{C} 2$ 电极在不同电流密度下的比电容(a)和倍率 特性(b)曲线

Fig. 8 Specific capacitance (a) and rate performance (b) for $\mathrm{C} 1$ and $\mathrm{C} 2$ electrodes at different current densities
这主要归因于该材料具有较高的比表面积和多级 的孔道结构，这样的结构能够有效促进电极材料 在充放电过程中与电解液的充分接触，使其储存 更多的能量。

图 9 是活化前多孔炭 $(\mathrm{C} 1)$ 和活化后 $(\mathrm{C} 2)$ 多孔炭 电极材料在 $1 \mathrm{~mol} / \mathrm{L} \mathrm{H}_{2} \mathrm{SO}_{4}$ 溶液中、 $5 \mathrm{~A} / \mathrm{g}$ 电流密度 下的循环寿命和库伦效率图。由图 9 可见, 经过 8000 圈的充放电循环后, 活化前电极材料的比电容 从第 1 圈的 $181.2 \mathrm{~F} / \mathrm{g}$ 下降到第 8000 圈的 $131.4 \mathrm{~F} / \mathrm{g}$, 衰减了 $27.5 \%$, 同时库伦效率保持在 $100 \%$ 左右; 而 活化后电极材料的比电容从第 1 圈的 $335.2 \mathrm{~F} / \mathrm{g}$ 下降 到第 8000 圈的 $300.2 \mathrm{~F} / \mathrm{g}$, 衰减了 $10.4 \%$, 同时库伦 效率也保持在 $100 \%$ 左右。这些数据表明活化后炭 材料比活化前炭材料具有更高的比电容和更好的循 环稳定性。

\section{8 电极材料的阻抗测试}

图 10 为活化前多孔炭 $(\mathrm{C} 1)$ 和活化后多孔炭 $(\mathrm{C} 2)$ 电极材料循环 8000 圈前后开路电压下的交流阻抗 图, 电解液为 $1 \mathrm{~mol} / \mathrm{L} \mathrm{H}_{2} \mathrm{SO}_{4}$, 振幅为 $5 \mathrm{mV}$, 频率范 围为 $10^{-2} \sim 10^{5} \mathrm{~Hz}$, 图中高频区的曲线与实轴的截距 表示活性物质的内阻 ${ }^{[29]}$ 。通过对比，活化后炭电极 的内阻(循环前: $1.65 \Omega$; 循环后: $2.79 \Omega$ )要低于活化 前炭电极的内阻(循环前：3.91 $\Omega$; 循环后：6.47 $\Omega$ ), 且每个电极循环后的内阻均大于循环前的内阻。此 外，在高频区，活化前后电极材料的交流阻抗曲线 均表现为不规则的小圆弧，意味着电极表面和电解 质之间的界面传荷电阻很小; 在低频区, 活化后电 极材料的交流阻抗曲线斜率明显大于活化前电极材 料低频区的交流阻抗斜率，表明活化后电极材料具 有更好的电容特性 ${ }^{[30-31]}$ 。上述结果说明尽管长时间 的充放电会引起电极材料阻抗的增加, 但相比之下,

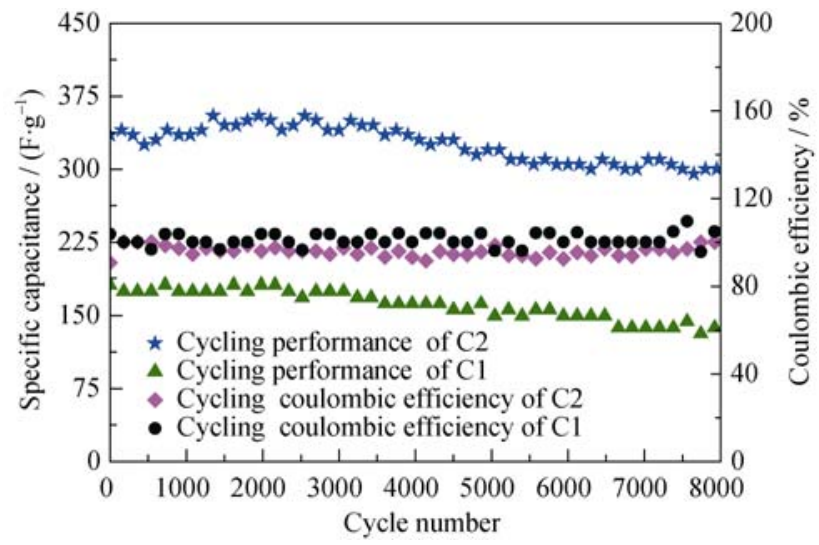

图 $9 \mathrm{C} 1$ 和 $\mathrm{C} 2$ 电极在 $5 \mathrm{~A} / \mathrm{g}$ 电流密度下的循环寿命图和库伦 效率图

Fig. 9 Cycling performance and coulombic efficiency of $\mathrm{C} 1$ and $\mathrm{C} 2$ electrodes at a current density of $5 \mathrm{~A} / \mathrm{g}$ 


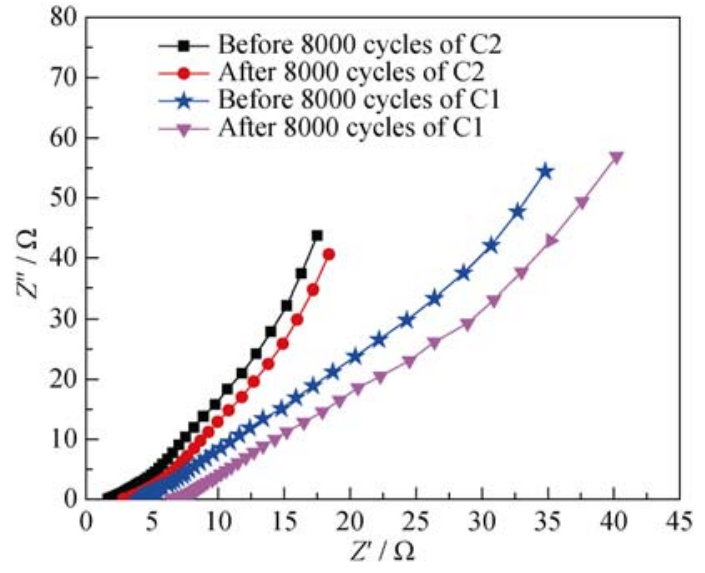

图 $10 \mathrm{C} 1$ 和 $\mathrm{C} 2$ 电极循环 8000 圈前后的交流阻抗曲线 Fig. 10 Nyquist plots before and after 8000 cycles of $\mathrm{C} 1$ and $\mathrm{C} 2$ electrodes

活化后的炭材料较活化前的炭材料具有更加优异的 电化学性能。

\section{3 结论}

以褐煤萃取物为前驱体, 以 $\mathrm{MgO}$ 为阻隔剂, $\mathrm{KOH}$ 为活化剂, 在 $\mathrm{N}_{2}$ 气氛保护下, 经过炭化-活化 过程, 获得了双电层电容器用多孔炭材料。该材料 较活化前炭材料显示了以下优良性能:

1) 微观结构呈现明显的多孔网状薄膜, 比表 面积高达 $1396 \mathrm{~m}^{2} / \mathrm{g}$, 而活化前炭材料比表面积仅为 $138.4 \mathrm{~m}^{2} / \mathrm{g}$ 。

2) 该多孔炭具有高的比电容。在 $1 \mathrm{~A} / \mathrm{g}$ 的电流 密度下的比电容为 $533 \mathrm{~F} / \mathrm{g}$, 而活化前炭材料的比电 容为 $366 \mathrm{~F} / \mathrm{g}$ 。

3) 该多孔炭具有良好的循环寿命。在电流密度 为 $5 \mathrm{~A} / \mathrm{g}$ 下循环 8000 圈后其电容保持率高达 $89.6 \%$, 而活化前炭材料的电容保持率为 $72.5 \%$ 。

以煤萃取物为炭源, 通过活化处理得到的多孔 炭材料具有良好的电化学性能, 该材料有望应用在 双电层电容器用电极材料上, 这将为煤的高值化利 用开辟新途径。

\section{参考文献:}

[1] WANG XIAO-QIN, ZHOU AN-NING, XIONG SHAN-XIN, et al. Preparation and capacitance property of anthracite/polyaniline composites. New Chemical Materials, 2014, 42(7): 196-198.

[2] WAN HOU-ZHAO, MIAO LING, XU KUI, et al. Manganese oxidebased electrode behavior as materials for electrochemical supercapacitors. Journal of Chemical Industry and Engineering, 2013, 64(3): 801-813.
[3] LU YUN, YUAN JIE, GONG YAN-KUN, et al. Study on effect of conductive agent on the performances of ruthenium oxide electrode materials. Journal of Functional Materials, 2014, 45(B12): $101-104$.

[4] KIERZEK K, FRACKOWIR E, LOTA G, et al. Electrochemical capacitors based on highly porous carbons prepared by $\mathrm{KOH}$ activation. Electrochim. Acta, 2004, 49(4): 515-523.

[5] HE XIAO-JUN, LEI JIANG-WEI, GENG YE-JING, et al. Preparation of microporous activated carbon and its electrochemical performance for electric double layer capacitor. J. Phys. Chem. Solids, 2009, 70(3/4): 738-744.

[6] ZHANG CHUAN-XIANG, LONG DONG-HUI, XING BAO-LIN, et al. The superior electrochemical performance of oxygen-rich activated carbons prepared from bituminous coal. Electrochem. Commun., 2008, 10(11): 1809-1811.

[7] LI XIAN, ASHIDA RYUICHI, MIURA KOUICHI. Preparation of high-grade carbonaceous materials having similar chemical and physical properties from various low-rank coals by degradative solvent extraction. Energy Fuels, 2012, 26(11): 6897-6904.

[8] SUN YE, WANG XING-JUN, FENG TING-TING, et al. Evaluation of coal extraction with supercritical carbon dioxide/1-methyl2-pyrrolidone mixed solvent. Energy Fuels, 2014, 28(2): 816-824.

[9] LI YI, ZHANG XIANG-PING, DONG HEI-FFENG, et al. Efficient extraction of direct coal liquefaction residue with the [bmim] Cl/NMP mixed solvent. RSC Adv., 2011, 1(8): 1579-1584.

[10] TAKANOHASHI TOSHIMASA, YANAGIDA TAKAYUKI, IINO MASASHI. Extraction and swelling of low-rank coals with various solvents at room temperature. Energy Fuels, 1996, 10(5): 1128-1132.

[11] XU GUO-ZHONG, WU HONG-YUN, GAO LI-JUAN, et al. Preparation and characterization of activated carbon with hierarchical pores based on semicoke powder by steam activation. Carbon Techniques, 2015, 31(2): 31-35.

[12] MAO AI-QIN, WANG HUA, TAN LING-HUA, et al. Research progress in characterization of functional groups on activated carbon. Applied Chemical Industry, 2011, 40(7): 1266-1270.

[13] LI CHUN-QI, TAKANOHASHI TOSHIMASA, SAITO IKUO. Coal dissolution by heat treatment at temperatures up to $300^{\circ} \mathrm{C}$ in $\mathrm{N}$-methyl-2-pyrrolidinone with addition of lithium halide. 2. Elucidation of mechanism by investigation of the structural changes of heat-treated coals. Energy Fuels, 2003, 17(3): 768-773.

[14] HU SI-XIAO, HSIEH YOU-LO. Preparation of activated carbon and silica particles from rice straw. ACS Sustainable Chem. Eng., 2014, 2(4): 726-734.

[15] ANDRES JOSE M, FERRANDO ANA C, FERRER PEDRO. Liquefaction of low-rank coals with hydriodic acid and microwaves. Energy Fuels, 1998, 12(3): 563-569. 
[16] MI HONG-YU, ZHOU JIA-PAN, ZHAO ZONG-BIN, et al. Block copolymer-guided fabrication of shuttle-like polyaniline nanoflowers with radiating whiskers for application in supercapacitors. RSC Adv., 2015, 5(2): 1016-1023.

[17] YUAN MEI-RONG, ZHAO FANG-HUI, LIU WEI-QIANG, et al. Preparation and properties of graphene for supercapacitor application. Journal of Functional Materials, 2013, 44(19): 2810-2813.

[18] WANG QIANG, CAO QI, WANG XIAN-YOU, et al. A high capacity carbon prepared from renewable chicken feather biopolymer for supercapacitors. J. Power Sources, 2013, 225: 101-107.

[19] SEVILLA MARTA, FUERTES ANTONIO B. Direct synthesis of highly porous interconnected carbon nanosheets and their application as high-performance supercapacitors. ACS Nano, 2014, 8(5): 5069-5078.

[20] ZHOU YING, WANG ZHI-CHAO, WANG CHUN-LEI, et al. Synthesis and properties of hierarchical macro-mesoporous carbon materials. Journal of Inorganic Materials, 2011, 26(2): 145-148.

[21] WANG LI-LI, XING RUI-GUANG, ZHANG BANG-WEN, et al. Preparation and electrochemical properties of functionalized graphene/polyaniline composite electrode materials. Acta Phy. -Chim. Sin., 2014, 30(9): 1659-1666.

[22] SHEN JIA-LI, YANG CHONG-YANG, LI XING-WEI, et al. High-performance asymmetric supercapacitor based on nanoarchitectured polyaniline/graphene/carbon nanotube and activated graphene electrodes. ACS Appl. Mater. Interfaces, 2013, 5(17): $8467-8476$.

[23] ZHU CHEN, ZHANG YUAN-LIANG, LIU HONG-TAO. Highly capacitance-enhanced activated carbons by oxidation-activation treatments for supercapacitor applications. Journal of Central
South University (Science and Technology), 2012, 43(12): 46384645.

[24] XIE YING-BO, ZHANG WEI-YAN, HANG RUI, et al. Comparisons of pore structure and electrochemical performances of carbons activated by $\mathrm{KOH}$ and $\mathrm{NaOH}$. Carbon Techniques, 2008, 27(2): 9-14.

[25] MENG QING-HAN, LIU LING, SONG HUAI-HE, et al. Electrochemical properties of carbon aerogels electrode for super-capacitor. Journal of Inorganic Materials, 2004, 19(3): 593-598.

[26] MIAO XIAO-LI, DENG ZHENG-HUA. Recent advances of electrode materials in electrochemical supercapacitors. Chinese Journal of Synthetic Chemistry, 2002, 2: 106-109.

[27] ZHANG ZHI-AN, DENG MEI-GEN, WANG BIN-HUA, et al. Electrochemical characterization of carbon black electrode for supercapacitor. Journal of Functional Materials, 2005, 36(2): 304-306.

[28] WANG KAI, ZHANG LI, GAO YUAN, et al. Template preparations for ordered mesoporous carbon and its electrochemical performances. Journal of Functional Materials, 2013, 44(1): 136-138.

[29] CONG HUAI-PING, REN XIAO-CHEN, WANG PING, et al. Flexible graphene-polyaniline composite paper for high-performance supercapacitor. Energy Environ. Sci., 2013, 6: 1185-1191.

[30] PROBSTLE H, SCHMITT C, FRICKE J. Button cell supercapacitors with monolithic carbon aerogels. J. Power Sources, 2002, 105(2): 189-194.

[31] SENTHILKUMAR S T, KALAI SELVAN R, MELO J S, et al. Performance solid-state electric double layer capacitor from redox mediated gel polymer electrolyte and renewable tamarind fruit shell derived porous carbon. ACS Appl. Mater. Interfaces, 2013, 5(21): 10541-10550. 